Reply to Comment on 'An educational path for the magnetic vector potential and its physical implications'

To cite this article: S Barbieri et al 2014 Eur. J. Phys. 35028002

- Comment on 'An educational path for the
$\frac{\text { magnetic vector potential and its physical }}{\text { implications' }}$
José A Heras
- An educational path for the magnetic
$\frac{\text { vector potential and its physical }}{\text { implications }}$
S Barbieri, M Cavinato and M Giliberti
- Corrigendum: An educational path for the
$\frac{\text { magnetic vector potential and its physical }}{\text { implications }}$
S Barbieri, M Cavinato and M Giliberti

View the article online for updates and enhancements. 


\title{
Reply to Comment on 'An educational path for the magnetic vector potential and its physical implications'
}

\author{
S Barbieri ${ }^{1}$, M Cavinato $^{2}$ and M Giliberti ${ }^{2}$ \\ ${ }^{1}$ Dipartimento di Fisica e Technologie Relative, Università degli Studi di Palermo, \\ Palermo, Italy \\ ${ }^{2}$ Dipartimento di Fisica, Università degli Studi di Milano, Milano, Italy \\ E-mail: marco.giliberti@unimi.it
}

Received 10 October 2013, revised 18 November 2013

Accepted for publication 25 November 2013

Published 17 January 2014

\begin{abstract}
In this reply we respond to the comment made by Heras on our paper (Barbieri et al 2013 Eur. J. Phys. 34 1209), in which we presented an educational path on the magnetic vector potential $\mathbf{A}$, aimed at undergraduate students and preservice physics teachers.
\end{abstract}

Keywords: vector potential, electromagnetic field, education

Our paper [1] has been commented on by Heras [2]. Starting from the initial consideration that our equation (3) 'is incorrect', he argues that our approach 'involves several inconsistencies' and therefore he reaches the conclusion that our educational path is 'unattractive from a pedagogical point of view'. In this reply we respond to the criticisms given by Heras.

Concerning point (a) of [2], we agree that equation (3) of [1] is incorrect since, starting from the Ampère-Maxwell law and the solenoidality of the magnetic field $\mathbf{B}$, one gets:

$$
\mathbf{B}(\mathbf{r}, t)=\frac{\mu_{0}}{4 \pi} \int_{V^{\prime}} \frac{\left[\mathbf{J}\left(\mathbf{r}^{\prime}, t\right)+\varepsilon_{0} \frac{\partial \mathbf{E}\left(\mathbf{r}^{\prime}, t\right)}{\partial t}\right] \times \Delta \mathbf{r}}{(\Delta r)^{3}} \mathrm{~d} V^{\prime},
$$

that is, one obtains an expression for $\mathbf{B}$ in terms of the present time. We are grateful to Heras for this observation.

As concerns the problem raised by Heras in point (b) of [2], we erroneously wrote to 'neglect all the time derivatives multiplied by $1 / c$ ', while it is quite obvious that it is the times derivatives multiplied by $1 / c^{2}$ that can be disregarded, as can be clearly seen from the rest of our original paper.

In [1], we should have done better by providing some explanatory words for the replacement of the retarded time $t^{\prime}$ with the present time $t$ : we have considered the approximation $\Delta r / c \sim 0$ (see equation (4) of [1]), as in a typical laboratory situation. In any case, using the correct formula of equation (1), this approximation is no longer required. 
In the framework of a slowly varying time-dependent field approximation, the starting point of our educational path is (equation (5) of [1]):

$$
\mathbf{B}(\mathbf{r}, t)=\frac{\mu_{0}}{4 \pi} \int_{V^{\prime}} \frac{\mathbf{J}\left(\mathbf{r}^{\prime}, t\right) \times \Delta \mathbf{r}}{(\Delta r)^{3}} \mathrm{~d} V^{\prime}
$$

which is absolutely correct and can be easily obtained from equation (1). Therefore the criticism in point (a) raised by Heras [2] does not in any way affect our subsequent educational path.

From equation (2) we obtained, with only simple and exact calculations, the expression (equation (9) of [1]):

$$
\mathbf{B}(\mathbf{r}, t)=\nabla \times\left(\frac{\mu_{0}}{4 \pi} \int_{V^{\prime}} \frac{\mathbf{J}\left(\mathbf{r}^{\prime}, t\right)}{\Delta r} \mathrm{~d} V^{\prime}\right),
$$

from which we found it natural to introduce the vector

$$
\mathbf{A}(\mathbf{r}, t) \equiv \frac{\mu_{0}}{4 \pi} \int_{V^{\prime}} \frac{\mathbf{J}\left(\mathbf{r}^{\prime}, t\right)}{\Delta r} \mathrm{~d} V^{\prime} .
$$

This vector is a particular magnetic vector potential and, moreover, is the magnetic vector potential which our path naturally leads to for slowly varying fields.

Concerning point (c) of [2], it is surely correct that in the slowly varying field approximation $\nabla \cdot \mathbf{J}=0$, but, in deriving our equation $\nabla \cdot \mathbf{A}=0$, we did not implicitly assume $\nabla \cdot \mathbf{J}=0$ nor did we deduce anything incorrectly; at most one can say that we have been too lengthy, since we deleted the time derivatives multiplied by $\varepsilon_{0} \mu_{0}=1 / c^{2}$ only at the end of the calculations.

As concerns point (d), the mathematical considerations given by [2] are well known and diffusely analysed in the literature. Most textbooks start from fixing the Coulomb gauge $\nabla \cdot \mathbf{A}=0$, with only the goal of simplifying the wave equation written in terms of the potentials. Our approach is the reverse. In fact we arrived at an expression for $\mathbf{B}$ written as the curl of a quantity, which we called $\mathbf{A}$. Through the usual definition $\mathbf{B}=\nabla \times \mathbf{A}$, one immediately sees that the vector $\mathbf{A}$ of equation (4) is a possible magnetic vector potential. Moreover, it is also expressed in terms of a clear empirical referent (the conduction currents). Hence we have chosen it as the most effective vector potential from a pedagogical point of view. Once the conduction currents are known, $\mathbf{A}$ is univocally determined.

Obviously, the gauge transformations (equations (23) and (24) of [1]) imply that the vector A of equation (4) is not the only possible vector potential.

To conclude this reply, we would like to stress that our path also covers many other topics, such as:

- the analogies between the vector and the scalar potentials;

- the physical meaning of the magnetic vector potential;

- interesting parallelisms with mechanical situations and, last but not least,

- the possibilities that $\mathbf{A}$ offers to give a local and causal description which is clearer than that obtained with $\mathbf{B}$ alone.

Therefore, we are confident that our educational approach can really be appropriate for the introduction of the magnetic vector potential to undergraduate students and pre-service physics teachers.

\section{References}

[1] Barbieri S, Cavinato M and Giliberti M 2013 An educational path for the magnetic vector potential and its physical implications Eur. J. Phys. 34 1209-19

[2] Heras J A 2014 Comment on 'An educational path for the magnetic vector potential and its physical implications' Eur. J. Phys. 35028001 